\title{
P. SABLONNIÈRE
}

\section{Étude de l'équation de Fredholm au voisinage d'une borne critique}

RAIRO - Analyse numérique, tome 11, no 3 (1977), p. 287-305.

<http://www.numdam.org/item?id=M2AN_1977_11_3_287_0>

(C) AFCET, 1977, tous droits réservés.

L'accès aux archives de la revue «RAIRO - Analyse numérique » implique l'accord avec les conditions générales d'utilisation (http://www.numdam.org/ legal.php). Toute utilisation commerciale ou impression systématique est constitutive d'une infraction pénale. Toute copie ou impression de ce fichier doit contenir la présente mention de copyright.

\section{Numdam}




\title{
ÉTUDE DE L'ÉQUATION DE FREDHOLM AU VOISINAGE D'UNE BORNE CRITIQUE ( ${ }^{1}$ )
}

\author{
par P. SABLONNIÈRE $\left({ }^{2}\right)$ \\ Communique pat P G CIARLi
}

Resume - Une borne crittque de l'équation de Fredholm est une borne superleure de l'intégrale rendant singulier l'opérateur correspondant On étudie le comportement de la solution et des fonctions singultères au votsinage d'une borne crittque et l'on donne des systèmes différentiels permettant le calcul de ces divers éléments

\section{POSITION DU PROBLÈME ET NOTATIONS UTILISÉES}

1.1. L'objet de cette étude est la résolution de l'équation :

$$
\tau(x)=f(x)+\lambda \int_{a}^{b} K(x, s) \tau(s) d s
$$

et de l'équation conjuguée :

$$
\tau^{\prime}(y)=g(y)+\bar{\lambda} \int_{a}^{b} K^{\prime}(y, s) \tau^{\prime}(s) d s
$$

où

$$
K^{\prime}(x, y)=\overline{K(y, x)}, \quad K \in C[a, b]^{2}, \quad f \text { et } g \in C[a, b] \text { et } \lambda \in \mathbf{C} .
$$

La méthode que nous ut1lisons est la variation de la borne supérieure de l'intégrale. Elle apparaît chez Sobolev [11], Gohberg et Krein [3] dans des résultats théoriques, puis elle est reprise par Pouzet [5] et Broudiscou [6] pour la résolution numérique. Elle est utilisée également par Atkinson [1], Schumitzky et Wenska [9].

\footnotetext{
(1) Manuscrit reçu le 24 novembre 1976

(2) I U T Informatıque, Villeneuve d'Ascq
}

R A I R O Analyse Numérique/Numerical Analysıs, vol 11, n 3, 1977 
On associe à $(E)$ et $\left(E^{\prime}\right)$ les équations dépendant de $z \in[a, b]$ :

$$
\begin{gathered}
\tau(x, z)=f(x)+\lambda \int_{a}^{z} K(x, s) \tau(s, z) d s \\
\tau^{\prime}(y, z)=g(y)+\bar{\lambda} \int_{a}^{z} K^{\prime}(y, s) \tau^{\prime}(s, z) d s
\end{gathered}
$$

L'opérateur intégral $K(z)$ est associé à l'équation $E(z)$ et son conjugué $K^{\prime}(z)$ à l'équation $E^{\prime}(z)$. Le problème est de déterminer $\tau(x, b)$ solution de $E(b)$ à partir de $\tau(x, a)=f(x)$ au moyen d'un système différentiel en $z$. Lorsque, pour tout $z \in[a, b]$, l'opérateur $I-\lambda K(z)$ est inversible, son inverse est de la forme $I+\lambda \Gamma(z)$ où $\Gamma(z)$ est l'opérateur intégral dont le noyau $\gamma(x, y, z)$ vérifie l'équation ( $c f$. [3] p. 186).

$$
\left\{\begin{array}{l}
\frac{\partial}{\partial z} \gamma(x, y, z)=\lambda \gamma(x, z, z) \gamma(z, y, z) \\
\gamma(x, y, a)=K(x, y)
\end{array}\right.
$$

Dans ce cas, la solution $\tau(x, z)$ de $E(z)$ est unique et vérifie l'équation :

$$
\left\{\begin{array}{l}
\frac{\partial}{\partial z} \tau(x, z)=\lambda \gamma(x, z, z) \tau(z, z) \\
\tau(x, a)=f(x)
\end{array}\right.
$$

(on a des résultats analogues pour l'équation conjuguée).

En revanche, les équations (1) et (2) ne sont plus valables sur tout l'intervalle $[a, b]$ lorsqu'il existe au moins un $\left.\left.z_{0} \in\right] a, b\right]$ tei que $I-\lambda K\left(z_{0}\right)$ ne soit pas inversible. Quand un tel $z_{0}$ existe, $\lambda$ devient valeur singulière de l'opérateur $K\left(z_{0}\right)$ et la fonction $\gamma(x, y, z)$ tend vers l'infini lorsque $z$ tend vers $z_{0}$.

Nous proposons l'utilisation des déterminants de Fredholm pour franchir les singularités rencontrées dans la variation de $z$ entre $a$ et $b$. On obtient également des résultats intéressants sur les fonctions singulières de $K\left(z_{0}\right)$ et les conditions d'existence des solutions de $E\left(z_{0}\right)$.

\subsection{Notations utilisées}

$$
\begin{aligned}
\Delta_{p}(z)=[a, z]^{p}, \quad \Delta_{p}=\Delta_{p}(b) . \\
X_{n}=\left(x_{1}, \ldots, x_{n}\right) \in \Delta_{n} ; \quad X_{0}=\varnothing(\text { aucune composante }) \\
X_{n, k}=\left(x_{1}, \ldots, x_{k-1}, x_{k+1}, \ldots, x_{n}\right) \in \Delta_{n-1} \\
X_{n} S_{p}=\left(x_{1}, \ldots, x_{n}, s_{1}, \ldots, s_{p}\right) \in \Delta_{n} \times \Delta_{p}(z) \\
x X_{n}=\left(x, x_{1}, \ldots, x_{n}\right) \in \Delta_{n+1} \\
X_{p, n}^{*}=\left(x_{p+1}, \ldots, x_{n}\right) \in \Delta_{n-p} .
\end{aligned}
$$


Pour toute fonction $\phi \in C\left(\Delta_{2}\right)$, on désigne par $\phi\left[\dot{X}_{p}, \dot{Y}_{p}\right]$ la matrice d'éléments $\phi\left(x_{i}, y_{j}\right)(1 \leqslant i \leqslant p, 1 \leqslant j \leqslant p)$ et on pose $\phi\left(\dot{X}_{p}, \dot{Y}_{p}\right)=\operatorname{dét} \phi\left[\dot{X}_{p}, \dot{Y}_{p}\right]$. Plus généralement, si $A\left(x X_{n}, y Y_{n}, z\right)$ est considérée comme fonction de $x$ et $y$, $A\left[\dot{X}_{p} X_{n}, \dot{Y}_{p} Y_{n}, z\right]$ désigne la matrice d'éléments $A\left[x_{i} X_{n}, y_{j} Y_{n}, z\right]$ et $A\left(\dot{X}_{p} X_{n}, \dot{Y}_{p} Y_{n}, z\right)$ le déterminant (d'ordre $p$ ) de la matrice $A\left[\dot{X}_{p} X_{n}, \dot{Y}_{p} Y_{n}, z\right]$.

On pose $\theta_{0}(\phi)=1, \theta_{1}(x, y ; \phi)=\phi(x, y)$ et pour $n \geqslant 2$ :

$$
\theta_{n}\left(x X_{n-1}, y Y_{n-1} ; \phi\right)=\operatorname{dett}_{n}\left(\begin{array}{cc}
\phi(x, y) & K\left[x, \dot{Y}_{n-1}\right] \\
\phi\left[\dot{X}_{n-1}, y\right] & K\left[\dot{X}_{n-1}, \dot{Y}_{n-1}\right]
\end{array}\right)
$$

On définit alors les fonctions suivantes :

$$
T_{0}(z ; \phi)=1+\sum_{p \geqslant 1}(-1)^{p} \frac{\lambda^{p}}{p !} \int_{\Delta_{p}(z)} \theta_{p}\left(S_{p}, S_{p} ; \phi\right) d S_{p}
$$

et, pour $n \geqslant 1$ :

$$
\begin{gathered}
T_{n}\left(x X_{n-1}, y Y_{n-1}, z ; \phi\right)=\theta_{n}\left(x X_{n-1}, y Y_{n-1} ; \phi\right) \\
+\sum_{p \geqslant 1} \frac{(-\lambda)^{p}}{p !} \int_{\Delta_{p}(z)} \theta_{n+p}\left(x X_{n-1} S_{p}, y Y_{n-1} S_{p} ; \phi\right) d S_{p} \\
T_{n}^{\prime}\left(x X_{n-1}, y Y_{n-1}, z ; \phi\right)=\bar{T}_{n}\left(y Y_{n-1}, x X_{n-1}, z ; \phi^{\prime}\right) \\
T_{0}^{\prime}(z ; \phi)=\bar{T}_{0}\left(z ; \phi^{\prime}\right) \quad \text { avec } \phi^{\prime}(x, y)=\overline{\phi(y, x)}
\end{gathered}
$$

REMARQUe : Pour $n \geqslant 2$, les fonctions $T_{n}$ et $T_{n}^{\prime}$ sont invariantes par permutation des couples de variables $\left(x_{i}, y_{i}\right)$ et $\left(x_{j}, y_{j}\right)(i \neq j)$.

\section{DÉTERMINANTS DE FREDHOLM ET FONCTIONS GÉNÉRATRICES}

Si $M$ désigne un majorant commun de $|\phi|$ et de $|K|$ sur $\Delta_{2}$, le lemme de Hadamard ([7], p. 175) fournit la majoration : $\left|\theta_{n}\right| \leqslant n^{n / 2} M^{n}$. On en déduit que $\left|T_{n}\right|$ est majoré indépendamment de $z \in[a, b]$ par la série de terme général.

$$
u_{p}(n)=\frac{(b-a)^{p}|\lambda|^{p}}{p !}(n+p)^{\frac{n+p}{2}} M^{n+p} \quad(p \geqslant 0)
$$

Comme $u_{p+1}(n) / u_{p}(n)$ tend vers 0 quand $p \rightarrow+\infty$ (pour tout $n \geqslant 0$ ), on en déduit la convergence absolue et uniforme des séries $T_{n}$ et $T_{n}^{\prime}$ par rapport à $z \in[a, b]$ pour tout $\lambda \in \mathbf{C}$ fixé. 


\subsection{Déterminant et mineurs de Fredholm}

Quand $\phi=K, T_{0}(z ; K)=d(z)$ est le déterminant de Fredholm et pour $n \geqslant 1, T_{n}\left(x_{n} X_{n-1}, y_{n} Y_{n-1}, z, K\right)=D_{n}\left(X_{n}, Y_{n}, z\right)$ est le mineur d'ordre $n$ de l'opérateur intégral $K(z)$. Nous appelons borne critique associée à $\lambda \in C$ toute racine de $d(z)=0$. L'ensemble $B C(\lambda)$ de ces bornes critiques est donc un compact de $[a, b]$ que nous supposerons fini pour simplifier. (La caractérisation de cet ensemble est un problème ouvert). Si $z_{0} \in B C(\lambda)$. $\lambda$ est valeur singulière de $K\left(z_{0}\right)$ et $\bar{\lambda}$ valeur singulière de $K^{\prime}\left(z_{0}\right)$.

\subsection{Fonctions génératrices}

Quand $\phi(x, y)=f(x)$, on pose

$$
\begin{gathered}
T_{n}\left(x X_{n-1}, y Y_{n-1}, z ; f\right)=\Omega_{n}\left(x, X_{n-1}, Y_{n-1}, z\right) \\
\quad \text { pour } n \geqslant 2 \text { et } T_{1}(x, y, z ; f)=\omega(x, z) .
\end{gathered}
$$

De même, quand $\phi(x, y)=g(y)$, on pose

$$
T_{n}^{\prime}\left(x X_{n-1}, y Y_{n-1}, z ; g\right)=\Omega_{n}^{\prime}\left(y, X_{n-1}, Y_{n-1}, z\right)
$$

pour $n \geqslant 2$ et $T_{1}^{\prime}(x, y, z ; g)=\omega^{\prime}(y, z)$.

Les fonctions $\Omega_{n}$ et $\Omega_{n}^{\prime}$ permettent le calcul des solutions de $E(z)$ et $E^{\prime}(z)$ même dans le cas où $z \in B C(\lambda)$ : on les appelle pour cette raison fonctions génératrices des solutions.

Remarque : Tout résultat concernant $T_{n}^{\prime}$ est associé à un résultat concernant $T_{n}$. Nous n'énoncerons et démontrerons en général que les seconds, les premiers s'en déduisant au moyen de la relation (5).

\section{SYSTÈME DIFFÉRENTIEL INFINI VÉRIFIÉ PAR LES $T_{n}$}

Les fonctions $T_{n}$ définies par (3) et (4) ne sont pas indépendantes. Le premier résultat les concernant est fourni par le :

THÉORÈme 1: Les fonctions $T_{n}$ sont de classe $C^{1}$ en $z$ et vérifient le système différentiel infini suivant :

$$
(S I)\left\{\begin{array}{l}
\frac{\partial}{\partial z} T_{n}\left(x X_{n-1}, y Y_{n-1}, z ; \phi\right)=-\lambda T_{n+1}\left(x X_{n-1} z, y Y_{n-1} z, z ; \phi\right) \\
T_{n}\left(x X_{n-1}, y Y_{n-1}, a ; \phi\right)=\theta_{n}\left(x X_{n-z}, y Y_{n-1} ; \phi\right)
\end{array}\right.
$$


Démonstration : Étant donné la convergence normale des séries $T_{n}$, on peut dériver terme à terme par rapport à $z$ et on obtient :

$$
\begin{aligned}
& \frac{\partial}{\partial z} T_{n}\left(x X_{n-1}, y Y_{n-1}, z ; \phi\right)=-\lambda \theta_{n+1}\left(x X_{n-1} z, y Y_{n-1} z ; \phi\right) \\
&+\sum_{p \geqslant 2} \frac{(-\lambda)^{p}}{p !} \frac{\partial}{\partial z} \int_{\Delta_{p}(z)} \theta_{n+p}\left(x X_{n-1} S_{p}, y Y_{n-1} S_{p} ; \phi\right) d S_{p}
\end{aligned}
$$

Comme les fonctions $\theta_{n+p}$ sont invariantes par permutation des couples $\left(s_{i}, s_{i}\right)$ et $\left(s_{j}, s_{j}\right)$, on a :

$$
\begin{aligned}
\frac{\partial}{\partial z} \int_{\Delta_{p}(z)} \theta_{n+p}\left(x X_{n-1} S_{p},\right. & \left.y Y_{n-1} S_{p} ; \phi\right) d S_{p} \\
& =p \int_{\Delta_{p-1}(z)} \theta_{n+p}\left(x X_{n-1} z S_{p-1}, y Y_{n-1} z S_{p-1} ; \phi\right) d S_{p-1}
\end{aligned}
$$

d'où l'on déduit immédiatement le système (SI).

Considérons maintenant l'ensemble des suites $\left\{T_{n}\right\}_{n \geqslant 0}$ de fonctions définies respectivement sur $\Delta_{2 n} \times \Delta_{1}$, continues sur leur domaine par rapport à l'ensemble de leurs variables, de classe $C^{1}$ en $z$ sur $\Delta_{1}$ (la dérivée par rapport à $z$ étant également globalement continue). Soit $m_{n}$ un majorant de $\left|T_{n}\right|$ sur son domaine. Nous définissons l'ensemble $E$ des suites $\left\{T_{n}\right\}$ ci-dessus vérifiant de plus la propriété : $(P)$ La série entière $\sum_{p \geqslant 0} m_{p} \frac{x^{p}}{p !}$ a un rayon de convergence infini.

Ceci entraîne que, pour tout $n \geqslant 0$, la série $\sum_{p \geqslant 0} m_{n+p} \frac{x^{p}}{p !}$ a également un rayon de convergence infini.

THÉORÈME 2 : Le système (SI) admet comme solution unique dans $E$ la suite $\left\{T_{n}\right\}_{n \geqslant 0}$ définie par (4), pour toute fonction $\phi \in C\left(\Delta_{2}\right)$ et tout $\lambda \in \mathbf{C}$ fixés.

Démonstration: Montrons d'abord que la suite $\left\{T_{n}\right\}_{n \geqslant 0}$ définie par (4) fait partie de l'ensemble $E$. Comme on l'a vu au $\S 2$, la fonction $\left|T_{n}\right|$ a pour majorant la série $m_{n}$ de terme général :

$$
u_{p}(n)=\frac{|\lambda|^{p}(b-a)^{p}}{p !}(n+p)^{\frac{n+p}{2}} M^{n+p}
$$

Si l'on pose

$$
\mu_{n}(x)=\sum_{p \geqslant 0}(n+p)^{\frac{n+p}{2}} \frac{x^{p}}{p !}
$$


on voit que

$$
m_{n}=M^{n} \mu_{n}(|\lambda| M(b-a))
$$

On voit facilement que $\mu_{n}^{\prime}(x)=\mu_{n+1}(x)(n \geqslant 0)$, d'où $\mu_{0}^{(p)}(x)=\mu_{p}(x)$ pour tout $p \geqslant 0$. On en déduit que, pour tous $\alpha$ et $\beta \in \mathbf{R}$, on a :

$$
\mu_{0}(\alpha+\beta)=\sum_{p \geqslant 0} \frac{\beta^{p}}{p !} \mu_{p}(\alpha)
$$

En particulier, si on pose $\alpha=|\lambda| M(b-a)$ et $\beta=M x$, on obtient :

$$
\begin{aligned}
\mu_{0}(|\lambda| M(b-a)+M x) & =\sum_{p \geqslant 0} \frac{x^{p}}{p !} M^{p} \mu_{p}(|\lambda| M(b-a)) \\
& =\sum_{p \geqslant 0} m_{p} \frac{x^{p}}{p !}<+\infty
\end{aligned}
$$

La propriété $(P)$ étant vérifiée, $\left\{T_{n}\right\}_{n \geqslant 0}$ fait partie de $E$.

Supposons qu'il existe dans $E$ une deuxième solution $\left\{V_{n}\right\}_{n \geqslant 0}$ du système (SI). L'intégration de ce système $p$ fois à partir de l'équation différentielle de $V_{n}$ donne :

$$
\begin{aligned}
V_{n}\left(x X_{n-1}, y Y_{n-1}, z ; \phi\right)=\theta_{n}\left(x X_{n-1}, y Y_{n-1} ; \phi\right) \\
\quad-\lambda \int_{a}^{z} \theta_{n+1}\left(x X_{n-1} s, y Y_{n-1} s ; \phi\right) d s+\ldots \\
+(-1)^{p} \frac{\lambda^{p}}{p !} \int_{\Delta_{p}(z)} \theta_{n+p}\left(x X_{n-1} S_{p}, y Y_{n-1} S_{p} ; \phi\right) d S_{p} \\
+(-1)^{p+1} \int_{\Delta_{1}(z) \times \Delta_{1}\left(s_{1}\right) \ldots \Delta_{1}\left(s_{p}\right)}^{V_{n+p+1}\left(x X_{n-1} S_{p+1}, y Y_{n-1} S_{p+1}, s_{p+1} ; \phi\right) d S_{p+1}}
\end{aligned}
$$

Si $v_{n}$ est un majorant de $\left|V_{n}\right|$ sur son domaine, le reste est majoré en module par

$$
\frac{|\lambda|^{p+1}}{(p+1) !}(b-a)^{p+1} v_{n+p+1}
$$

c'est le terme général de la série $\sum_{p \geqslant 0} \frac{x^{p}}{p !} v_{n+p}$ avec $x=|\lambda|(b-a)$, donc, il tend vers 0 quand $p \rightarrow+\infty$ et $V_{n}$ coïncide avec $T_{n}$, c.q.f.d.

En pratique, un tel système est inutilisable. Nous allons démontrer certaines propriétés des fonctions $T_{n}$ qui nous permettrons de nous ramener à un système différentiel fini. 


\section{EQUATIONS VÉRIFIÉES PAR LES $T_{n}$}

On donne maintenant les équations intégrales vérifiées par les fonctions $T_{n}$ ainsi que les expressions des $T_{n}$ en fonction des $T_{k}(1 \leqslant k \leqslant n-1)$ et des déterminants de Fredholm. Ces équations sont utilisées dans la suite pour l'obtention des systèmes différentiels permettant le calcul des solutions et des fonctions singulières éventuelles de $E(z)$.

\section{1. Équations intégrales}

Développons le déterminant $\theta_{n+p}\left(x X_{n-1} S_{p}, y Y_{n-1} S_{n} ; \phi\right)$ par rapport à la première ligne, on obtient :

$$
\begin{aligned}
\phi(x, y) K\left(X_{n-1} \dot{S}_{p}, Y_{n-1}\right. & \left.\dot{S}_{p}\right) \\
& +\sum_{k=1}^{n-1}(-1)^{k} K\left(x, y_{k}\right) \theta_{n+p-1}\left(X_{n-1} S_{p}, y Y_{n-1, k} S_{p} ; \phi\right) \\
& +\sum_{k=1}^{p}(-1)^{n+k-1} K\left(x, s_{k}\right) \theta_{n+p-1}\left(X_{n-1} S_{p}, y Y_{n-1} S_{p, k} ; \phi\right)
\end{aligned}
$$

En intégrant sur le domaine $\Delta_{p}(z)$, en multipliant $\operatorname{par} \frac{(-\lambda)^{p}}{p !}$ et en sommant de $p=0$ à $+\infty$, on obtient :

$$
\begin{aligned}
& T_{n}\left(x X_{n-1}, y Y_{n-1}, z ; \phi\right)=\phi(x, y) D_{n-1}\left(X_{n-1}, Y_{n-1}, z\right) \\
&+ \sum_{k=1}^{n-1}(-1)^{k} K\left(x, y_{k}\right) T_{n-1}\left(X_{n-1}, y Y_{n-1, k}, z ; \phi\right) \\
&+\lambda \int_{a}^{z} K(x, s) T_{n}\left(s X_{n-1}, y Y_{n-1}, z ; \phi\right) d s
\end{aligned}
$$

Développons $\theta_{n+p}$ par rapport à la première colonne :

$$
\begin{aligned}
\phi(x, y) K\left(X_{n-1} \dot{S}_{p}, Y_{n-1}\right. & \left.\dot{S}_{p}\right) \\
& +\sum_{k=1}^{n-1}(-1)^{k} \phi\left(x_{k}, y\right) K_{n+p-1}\left(x X_{n-1 k} S_{p}, Y_{n-1} S_{p}\right) \\
& +\sum_{k=1}^{p}(-1)^{n+k-1} \phi\left(s_{k}, y\right) K_{n+p-1}\left(x X_{n-1} S_{p, k}, Y_{n-1} S_{p}\right)
\end{aligned}
$$


En intégrant sur $\Delta_{p}(z)$ par rapport à $S_{p}$, en multipliant par $\frac{(-\lambda)^{p}}{p !}$ et en sommant de $p=0$ à $+\infty$, on obtient :

$$
\begin{aligned}
T_{n}\left(x X_{n-1}, y Y_{n-1}, z ; \phi\right) & =\phi(x, y) D_{n-1}\left(X_{n-1}, Y_{n-1}, z\right) \\
& +\sum_{k=1}^{n-1}(-1)^{k} \phi\left(x_{k}, y\right) D_{n-1}\left(x X_{n-1 k}, Y_{n-1}, z\right) \\
& +\lambda \int_{a}^{z} D_{n}\left(x X_{n-1}, s Y_{n-1}, z\right) \phi(s, y) d s
\end{aligned}
$$

\subsection{Expressions des $T_{n}$ sous forme de déterminants}

THÉORÈME $3:$ Pour tout $z \in[a, b]$ et tout $n \geqslant 2$, on $a$ :

$$
\begin{aligned}
&(d(z))^{n-1} T_{n}\left(x X_{n-1}, y Y_{n-1}, z ; \phi\right) \\
&=\operatorname{dét}_{n}\left(\begin{array}{cc}
T_{1}(x, y, z ; \phi) & D_{1}\left[x, \dot{Y}_{n-1}, z\right] \\
T_{1}\left[\dot{X}_{n-1}, y, z ; \phi\right] & D_{1}\left[\dot{X}_{n-1}, \dot{Y}_{n-1}, z\right]
\end{array}\right)
\end{aligned}
$$

Plus généralement, pour $n \geqslant 3$ et $1 \leqslant p \leqslant n-1$ :

$$
\begin{aligned}
& \left(D_{p}\left(X_{p}, Y_{p}, z\right)\right)^{n-p-1} T_{n}\left(x X_{n-1}, y Y_{n-1}, z ; \phi\right) \\
& =\operatorname{det}_{n-p}\left(\begin{array}{cc}
T_{p+1}\left(x X_{p}, y Y_{p}, z ; \phi\right) & D_{p+1}\left[x X_{p}, Y_{p} \dot{Y}_{p n-1}^{*}, z\right] \\
T_{p+1}\left[X_{p} \dot{X}_{p n-1}^{*}, y Y_{p}, z ; \phi\right] & D_{p+1}\left[X_{p} \dot{X}_{p n-1}^{*}, Y_{p} \dot{Y}_{p n-1}^{*}, z\right]
\end{array}\right)
\end{aligned}
$$

Démonstration : Pour $n=2$, la relation (8) s'écrit :

$$
d(z) T_{2}\left(x x_{1}, y y_{1}, z ; \phi\right)=\left|\begin{array}{cc}
T_{1}(x, y, z ; \phi) & D_{1}\left(x, y_{1}, z\right) \\
T_{1}\left(x_{1}, y, z ; \phi\right) & D_{1}\left(x_{1}, y_{1}, z\right)
\end{array}\right|
$$

La fonction $T_{2}$ étant solution de l'équation intégrale (6) avec $n=2$, on a, pour $z \notin B C(\lambda)$ :

$$
\begin{aligned}
& d(z) T_{2}\left(x x_{1}, y y_{1}, z ; \phi\right) \\
& =D_{1}\left(x_{1}, y_{1}, z\right)\left[\phi(x, y) d(z)+\lambda \int_{a}^{z} D_{1}(x, s, z) \phi(s, y) d s\right] \\
& \quad-T_{1}\left(x_{1}, y, z ; \phi\right)\left[K\left(x, y_{1}\right) d(z)+\lambda \int_{a}^{z} D_{1}(x, s, z) K\left(s, y_{1}\right) d s\right]
\end{aligned}
$$


Les crochets étant respectivement égaux à $T_{1}(x, y, z ; \phi)$ et à $D_{1}\left(x, y_{1}, z\right)$ en vertu de (6), on obtient bien la relation (10). Comme les fonctions $T_{n}$ sont continues par rapport à $z$ et que $B C(\lambda)$ est supposé fini, la relation (10) se prolonge à $z \in B C(\lambda)$ et dans ce cas, le déterminant du second membre est nul.

Supposons la relation (8) vraie, pour $n-1$ : on obtient, à partir de l'équation intégrale (6) :

$$
\begin{aligned}
d(z) T_{n}\left(x X_{n-1}, y Y_{n-1}, z ; \phi\right)= & D_{n-1}\left(X_{n-1}, Y_{n-1}, z\right) T_{1}(x, y, z ; \phi) \\
& +\sum_{k=1}^{n-1} T_{n-1}\left(X_{n-1}, y Y_{n-1 . k}, z ; \phi\right) D_{1}\left(x, y_{k}, z\right)
\end{aligned}
$$

En multipliant par $(d(z))^{n-2}$ et en utilisant l'hypothèse de récurrence, le second membre est le développement par rapport à la première ligne du déterminant d'ordre $n$ de (8).

Pour la relation (9), on utilise l'identité de Sylvester sur les déterminants (cf. Gantmacher [2] p. 32-33) : si $\boldsymbol{A}$ est une matrice carrée d'ordre $n$;

soit $A\left(\begin{array}{lll}i_{1} i_{2} & \ldots & i_{k} \\ j_{1} j_{2} & \ldots & j_{k}\end{array}\right)$ le déterminant d'ordre $k$ extrait de $A$ en prenant les lignes $i_{l}$ et les colonnes $j_{l}(1 \leqslant l \leqslant k)$. Soit $B_{p}$ la matrice d'éléments :

$$
b_{i k}=A\left(\begin{array}{cccc}
1 & 2 \ldots p & i \\
1 & 2 \ldots p & k
\end{array}\right) \quad(i, k=p+1, \ldots, n)
$$

L'identité de Sylvester s'écrit :

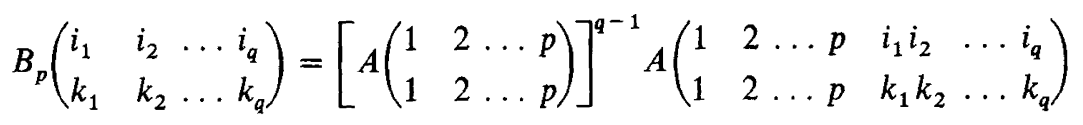

pour $p \leqslant i_{1} \leqslant \ldots \leqslant i_{q} \leqslant n$ et $p \leqslant k_{1} \leqslant \ldots \leqslant k_{q} \leqslant n$.

On prend pour $A$ la matrice suivante :

$$
A=\left(\begin{array}{cc}
T_{1}\left[\dot{X}_{n-1}, y, z ; \phi\right] & D_{1}\left[\dot{X}_{n-1}, \dot{Y}_{n-1}, z\right] \\
T_{1}(x, y, z ; \phi) & D_{1}\left[x, \dot{Y}_{n-1}, z\right]
\end{array}\right)
$$

On utilise (11) avec $q=n-p, i_{1}=k_{1}=p+1, \ldots, i_{q}=k_{q}=n$. En se servant de (8) pour $T_{n}$ et $D_{n}$, on trouve (9), c.q.f.d.

5. SOLUTION DE $E(z)$ QUAND $z \notin B C(\lambda)$

5.1. On sait que la solution de $E(z)$ est donnée par :

$$
\tau(x, z)=f(x)+\lambda \int_{a}^{z} \frac{D_{1}(x, s, z)}{d(z)} f(s) d s
$$


Or, l'équation (7), appliquée à $\phi(x, y)=f(x)$ et $n=1$, donne :

$$
\omega(x, z)=d(z) f(x)+\lambda \int_{a}^{z} D_{1}(x, s, z) f(s) d s .
$$

On en déduit :

$$
\forall z \notin B C(\lambda) \quad \tau(x, z)=\frac{\omega(x, z)}{d(z)}
$$

5.2. Système différentiel donnant $d, D_{1}$ et $\omega$

Du système (SI) (théorème 1), appliqué à $\phi=K$ et $\phi=f$, on tire, en utilisant la relation (8) :

$$
\begin{aligned}
d^{\prime}(z) & =-\lambda D_{1}(z, z, z) \\
d(z) \frac{\partial}{\partial z} D_{1}(x, y, z) & =\lambda\left|\begin{array}{ll}
D_{1}(x, z, z) & D_{1}(x, y, z) \\
D_{1}(z, z, z) & D_{1}(z, y, z)
\end{array}\right| \\
d(z) \frac{\partial}{\partial z} \omega(x, z) & =\lambda\left|\begin{array}{ll}
D_{1}(x, z, z) & \omega(x, z) \\
D_{1}(z, z, z) & \omega(z, z)
\end{array}\right|
\end{aligned}
$$

avec $d(a)=1, D_{1}(x, y, a)=K(x, y)$ et $\omega(x, a)=f(x)$.

Le système ci-dessus, que nous appellerons $\left(S F_{0}\right)$ dans la suite, permet le calcul de $d, D_{1}$ et $\omega$ (donc de $\tau$ ) sur tout intervalle où la fonction $d(z)$ ne s'annule pas (en particulier au voisinage de $a$ puisque $d(a)=1$ ). On en déduit que le noyau résolvant partiel

$$
\Gamma(x, y, z)=\frac{D_{1}(x, y, z)}{d(z)} \quad \text { et la solution } \quad \tau(x, z)=\frac{\omega(x, z)}{d(z)}
$$

vérifient les équations (1) et (2), données au paragraphe (1.1) sur tout intervalle ne contenant pas de borne critique.

\subsection{Existence et unicité de la solution du système $\left(S F_{0}\right)$}

Considérons un intervalle $[\alpha, \beta]$ de $[a, b]$ ne contenant pas de borne critique. Sur cet intervalle, le système $\left(S F_{0}\right)$ admet au moins une solution : les fonctions $d, D_{1}$ et $\omega$ construites plus haut.

Soit $E_{0}[\alpha, \beta]$ l'ensemble des triplets $(\delta, \mathscr{D}, \varpi)$ de fonctions définies et continues respectivement $\operatorname{sur}[\alpha, \beta], \Delta_{2} \times[\alpha, \beta]$ et $\Delta_{1} \times[\alpha, \beta]$, possédant chacune une dérivée partielle en $z$ continue sur son domaine de définition, vérifiant les conditions initiales $\delta(\alpha)=d(\alpha), \mathscr{D}(x, y, \alpha)=D(x, y, \alpha)$ et $\varpi(x, \alpha)=\omega(x, a)$, et telles que $\delta(z)$ ne s'annule pas sur $[\alpha, \beta]$. 
THÉORÈME $4:$ Le système $\left(S F_{0}\right)$ admet comme solution unique dans $E_{0}$ le triplet $\left(d, D_{1}, \omega\right)$.

Démonstration : $\mathrm{Si}(\delta, \mathscr{D}, \bar{\omega})$ vérifie les conditions ci-dessus, désignons par $m>0$ un minorant commun de $|\delta|$ et $|d|$ et $M$ un majorant commun de $\left|D_{1}\right|,|\mathscr{D}|,|d|$ et $|\delta|$ sur leurs domaines respectifs. On obtient alors :

$$
|\delta(z)-d(z)| \leqslant|\lambda| \int_{a}^{z}\left|\mathscr{D}(s, s, s)-D_{1}(s, s, s)\right| d s
$$

et

$$
\begin{aligned}
& \left|\mathscr{D}(x, y, z)-D_{1}(x, y, z)\right| \leqslant|\lambda| \int_{a}^{z} \mid \frac{1}{\delta(s)}(\mathscr{D}(x, y, s) \mathscr{D}(s, s, s) \\
& -\mathscr{D}(x, s, s) \mathscr{D}(s, y, s))-\frac{1}{d(s)}\left(D_{1}(x, y, s) D_{1}(s, s, s)-D_{1}(x, s, s) D_{1}(s, y, s)\right) \mid d s
\end{aligned}
$$

Posons $\mu(s)=\max _{(x, y) \in \Delta_{2}}|\mathscr{D}(x, y, s)-D(x, y, s)|$ pour $s \in[\alpha, \beta]$. On obtient alors, à partir de la majoration précédente :

$$
0 \leqslant \mu(z) \leqslant \frac{2|\lambda| M^{2}}{m^{2}}(|\lambda|(\beta-\alpha)+2) \int_{a}^{z} \mu(s) d s .
$$

en utilisant $|\delta(s)-d(s)| \leqslant|\lambda| \int_{a}^{z} \mu(u) d u$, puis

$$
\int_{a}^{z}|\delta(s)-d(s)| d s \leqslant|\lambda|(\beta-\alpha) \int_{a}^{z} \mu(u) d u, \forall z \in[\alpha, \beta] .
$$

L'inégalité $\left({ }^{*}\right)$ est du type $0 \leqslant \mu(z) \leqslant C \int_{a}^{=} \mu(s) d s$, elle implique donc $\mu(z)=0$ pour tout $z \in[\alpha, \beta]$, d'où $\mathscr{D}=D$, puis $\delta=d$ et enfin $\varpi=\omega$ par un raisonnement analogue à partir de (17).

Remarques : 1) Si l'on sait que $B C(\lambda)$ est vide, il est préférable d'utiliser (1) et (2) plutôt que $\left(S F_{0}\right)$ sur $[a, b]$.

2) Si l'on ne connaît pas la structure de $B C(\lambda)$, on doit utiliser $\left(S F_{0}\right)$ pour calculer $d(z)$.

3) D'autre part, il est plus simple, d'un point de vue numérique, d'intégrer uniquement (15) et (16) pour calculer $d$ et $D_{1}$ et d'utiliser (13) pour calculer $\omega$. 


\section{SOLUTION DE $E(z)$ AU VOISINAGE D'UNE BORNE CRITIQUE}

Nous supposons que $z_{0}$ est un point isolé de $B C(\lambda)$ et nous cherchons à étudier le comportement de l'équation $E(z)$ au voisinage de $z_{0}$ : rappelons que $\lambda$ est valeur singulière de l'opérateur $K\left(z_{0}\right)$; donc $d\left(z_{0}\right)=0$.

\subsection{Résultats généraux}

Si $\lambda$ est valeur singulière d'ordre $N$ de l'öpérateur $K\left(z_{0}\right)$, on sait qu'il existe $n \leqslant N$ ( $n$ est l'indice de défaut ou multiplicité propre de $\lambda$ ) et $\left(\stackrel{\circ}{X}_{n}, \stackrel{\circ}{Y}_{n}\right) \in \Delta_{2 n}$ tels que $D_{n}\left(\AA_{n}, \stackrel{\circ}{Y}_{n}, z_{0}\right) \neq 0$. Des solutions de base des équations homogènes associées à $E\left(z_{0}\right)$ et $E^{\prime}\left(z_{0}\right)$ sont données par :

$$
\begin{array}{ll}
\Phi_{i}(x)=D_{n}\left(x \dot{X}_{n, i}, \ddot{Y}_{n}, z_{0}\right) & (1 \leqslant i \leqslant n) \\
\Psi_{j}(y)=\bar{D}_{n}\left(\dot{X}_{n}, y \stackrel{\ominus}{Y}_{n, j}, z_{0}\right) & (1 \leqslant j \leqslant n)
\end{array}
$$

Ce sont les fonctions singulières de $K\left(z_{0}\right)$ et $K^{\prime}\left(z_{0}\right)$ respectivement. Les fonctions $D_{p}\left(X_{p}, Y_{p}, z_{0}\right)$ étant identiquement nulles pour $p<N$, on en déduit que les fonctions $\Omega_{p}$ et $\Omega_{p}^{\prime}$ sont également identiquement nulles pour $p<n$. L'équation (7) se simplifie et il reste :

$$
\begin{aligned}
& \Omega_{n}\left(x, X_{n-1}, Y_{n-1}, z_{0}\right)=\lambda \int_{a}^{z_{0}} D_{n}\left(x X_{n-1}, s Y_{n-1}, z_{0}\right) f(s) d s \\
& \Omega_{n}^{\prime}\left(y, X_{n-1}, Y_{n-1}, z_{0}\right)=\lambda \int_{a}^{z_{n}} \bar{D}_{n}\left(s X_{n-1}, y Y_{n-1}, z_{0}\right) g(s) d s .
\end{aligned}
$$

Pour que les équations complètes $E\left(z_{0}\right)$ et $E^{\prime}\left(z_{0}\right)$ admettent une solution particulière, il faut et il suffit que l'on ait respectivement :

$$
\begin{gathered}
\int_{a}^{z_{0}} \Psi_{j}(s) \overline{f(s)} d s=0 \quad(1 \leqslant j \leqslant n) \\
\int_{a}^{z_{0}} \Phi_{i}(s) \overline{g(s)} d s=0 \quad(1 \leqslant i \leqslant n)
\end{gathered}
$$

En comparant (19 et (18 ), puis (19') et (18), on voit que ces conditions équivalent respectivement à :

$$
\Omega_{n}\left(\stackrel{\circ}{x}_{j}, \hat{X}_{n, j}, \stackrel{Y}{n, j}_{n}, z_{0}\right)=0 \quad(1 \leqslant j \leqslant n)
$$

et

$$
\Omega_{n}^{\prime}\left(\stackrel{\circ}{i}_{i}, \stackrel{\circ}{X}_{n, i}, \stackrel{\circ}{Y}_{n, i}, z_{0}\right)=0 \quad(1 \leqslant i \leqslant n)
$$


Si ces conditions sont satisfaites, l'équation (9) donne :

$$
\begin{aligned}
O= & D_{n-1}\left(X_{n-1}, Y_{n-1}, z_{0}\right) \Omega_{n+1}\left(x, X_{n}, Y_{n}, z_{0}\right) \\
=\Omega_{n}\left(x, X_{n-1}, Y_{n-1}, z_{0}\right) D_{n}\left(X_{n}, Y_{n}, z_{0}\right) & \begin{aligned}
& \\
& \quad-\Omega_{n}\left(x_{n}, X_{n-1}, Y_{n-1}, z_{0}\right) D_{n}\left(x X_{n-1}, Y_{n}, z_{0}\right)
\end{aligned}
\end{aligned}
$$

En prenant : $X_{n-1}=\stackrel{\circ}{X}_{n, j}, Y_{n-1}=\stackrel{\circ}{Y}_{n, j}$ et $x_{n}=\stackrel{\circ}{x}_{j}$, on obtient :

$$
\Omega_{n}\left(x, \stackrel{\circ}{X}_{n, j}, \stackrel{\circ}{Y}_{n, j}, z_{0}\right)=\Omega_{n}\left(\stackrel{\circ}{x}_{j}, \stackrel{\circ}{X}_{n, j}, \stackrel{\circ}{Y}_{n, j}, z_{0}\right) \frac{D_{n}\left(x, \stackrel{\circ}{X}_{n . j}, \stackrel{\circ}{Y}_{n}, z_{0}\right)}{D_{n}\left(\stackrel{\circ}{X}_{n}, \stackrel{\circ}{Y}_{n}, z_{0}\right)}
$$

On en déduit immédiatement que toutes les fonctions $\Omega_{n}\left(x, \stackrel{\circ}{X}_{n, j}, \stackrel{\circ}{Y}_{n, j}, z_{0}\right)$ $(1 \leqslant j \leqslant n)$ et par un raisonnement analogue, les fonctions $\Omega_{n}^{\prime}\left(y, \stackrel{\circ}{X}_{n, i}, \stackrel{\circ}{Y}_{n-i}, z_{0}\right)$ (pour $1 \leqslant i \leqslant n$ ) sont identiquement nulles.

Une solution particulière de l'équation $E\left(z_{0}\right)$ est donnée par :

$$
\tilde{S}(x)=f(x)+\frac{\lambda}{D_{n}\left(\stackrel{\circ}{X}_{n}, \stackrel{\circ}{Y}_{n}, z_{0}\right)} \int_{a}^{z_{0}} D_{n+1}\left(x \stackrel{\circ}{X}_{n}, s \stackrel{\circ}{Y}_{n}, z_{0}\right) f(s) d s .
$$

Or on déduit de (7) que :

$$
\begin{aligned}
\Omega_{n+1}\left(x, \stackrel{\circ}{X}_{n}, \stackrel{\circ}{Y}_{n}, z_{0}\right)=D_{n}\left(\stackrel{\circ}{X}_{n}, \stackrel{\circ}{Y}_{n}, z_{0}\right) f(x) & +\sum_{k=1}^{n}(-1)^{k} f\left(x_{k}\right) \Phi_{k}(x) \\
& +\lambda \int_{a}^{z_{0}} D_{n+1}\left(x \stackrel{\circ}{X}_{n}, s \stackrel{\circ}{Y}_{n}, z_{0}\right) f(s) d s
\end{aligned}
$$

Par conséquent, la fonction :

$$
S(x)=\frac{\Omega_{n+1}\left(x, \stackrel{\circ}{X}_{n}, \stackrel{\circ}{Y}_{n}, z_{0}\right)}{D_{n}\left(\stackrel{\circ}{X}_{n}, \stackrel{\circ}{Y}_{n}, z_{0}\right)}=\tilde{S}(x)+\sum_{k=1}^{n}(-1)^{k} \frac{f\left(x_{k}\right)}{D_{n}\left(\stackrel{\circ}{X}_{n}, \dot{\circ}_{n}, z_{0}\right)} \Phi_{k}(x)
$$

est également solution particulière de $E\left(z_{0}\right)$. De même, la fonction

$$
S^{\prime}(y)=\frac{\Omega_{n+1}^{\prime}\left(y, \stackrel{\circ}{X}_{n}, \stackrel{\circ}{Y}_{n}, z_{0}\right)}{\bar{D}_{n}\left(\dot{\circ}_{n}, \stackrel{\circ}{Y}_{n}, z_{0}\right)}
$$

est solution de $E^{\prime}\left(z_{0}\right)$.

Nous pouvons énoncer le :

THÉORÈME 5 : Si $\lambda$ (resp. $\bar{\lambda}$ ) est valeur singulière de l'opérateur $K\left(z_{0}\right)$ (resp. $K^{\prime}\left(z_{0}\right)$ ), une condition nécessaire et suffisante pour que l'équation $E\left(z_{0}\right)$ (resp. $E^{\prime}\left(z_{0}\right)$ ) ait une solution est que toutes les fonctions $\Omega_{n}\left(x, \stackrel{\circ}{X}_{n, i}, \stackrel{\circ}{Y}_{n, i}, z_{0}\right)$ 
$(1 \leqslant i \leqslant n)$ soient identiquement nulles (resp. les fonctions $\left.\Omega_{n}^{\prime}\left(y, X_{n, i}, Y_{n, i}, z_{0}\right)\right)$. Une solution particulière de $E\left(z_{0}\right)$ (resp. de $E^{\prime}\left(z_{0}\right)$ ) est alors donnée par :

$$
\begin{gathered}
S(x)=\frac{\Omega_{n+1}\left(x, \dot{X}_{n}, \ddot{Y}_{n}, z_{0}\right)}{D_{n}\left(\dot{\circ}_{n}, \stackrel{\circ}{Y}_{n}, z_{0}\right)} \\
\text { (resp. } \left.S^{\prime}(y)\right)=\frac{\Omega_{n+1}^{\prime}\left(\dot{y}, \stackrel{\circ}{X}_{n}, \grave{\leftrightarrow}_{n}, z_{0}\right)}{D_{n}\left(\stackrel{\circ}{X}_{n}, \stackrel{\circ}{Y}_{n}, z_{0}\right)} .
\end{gathered}
$$

\subsection{Cas où $z_{0}$ est racine simple de $d(z)$}

6.2.1. Cette condition équivaut à $d^{\prime}\left(z_{0}\right) \neq 0$, ou encore $D_{1}\left(z_{0}, z_{0}, z_{0}\right) \neq 0$. Elle entraîne que le noyau de l'opérateur $I-\lambda K\left(z_{0}\right)$ est de dimension 1 (une seule fonction singulière de base). L'exemple $K(t, s)=3(1-t)(1-s)$, donné dans [9], où $d(z)=(1-z)^{3}$ pour $\lambda=1$, montre que la réciproque est fausse. On peut caractériser les bornes critiques simples par le :

THÉORÈME 6 : Une condition nécessaire et suffisante pour que $z_{0}$ soit borne critique simple est que le noyau de $I-\lambda K\left(z_{0}\right)$ soit de dimension 1 et que les fonctions singulières de $K\left(z_{0}\right)$ et $K^{\prime}\left(z_{0}\right)$ ne s'annulent pas au point $z_{0}$.

Démonstration : $\mathrm{Si} z_{0}$ est borne critique simple, le noyau de $I-\lambda K\left(z_{0}\right)$ est engendré par la fonction singulière $\phi(x)=D_{1}\left(x, z_{0}, z_{0}\right)$ et celui $I-\bar{\lambda} K^{\prime}\left(z_{0}\right)$ par $\psi(y)=\bar{D}_{1}\left(z_{0}, y, z_{0}\right):$ on voit immédiatement que

$$
\phi\left(z_{0}\right)=\bar{\psi}\left(z_{0}\right)=D_{1}\left(z_{0}, z_{0}, z_{0}\right) \neq 0 .
$$

Réciproquement, si le noyau de $I-\lambda K\left(z_{0}\right)$ est de dimension 1 , il existe $\left(x_{0}, y_{0}\right) \in \Delta_{2}$ tel que $D_{1}\left(x_{0}, y_{0}, z_{0}\right) \neq 0$ : les fonctions $\phi(x)=D_{1}\left(x, y_{0}, z_{0}\right)$ et $\psi(y)=\bar{D}_{1}\left(x_{0}, y, z_{0}\right)$ sont alors les fonctions singulières de $K\left(z_{0}\right)$ et $K^{\prime}\left(z_{0}\right)$ respectivement. La relation (10) donne, en $z=z_{0}$ (pour $\phi=K$ ) :

$$
D_{1}\left(x_{0}, y_{0}, z_{0}\right) D_{1}\left(z_{0}, z_{0}, z_{0}\right)=D_{1}\left(x_{0}, z_{0}, z_{0}\right) D_{1}\left(z_{0}, y_{0}, z_{0}\right)=\bar{\psi}\left(z_{0}\right) \phi\left(z_{0}\right)
$$

Comme le second membre et $D_{1}\left(x_{0}, y_{0}, z_{0}\right)$ sont non nuls par hypothèse, on en déduit $D_{1}\left(z_{0}, z_{0}, z_{0}\right) \neq 0$ et $z_{0}$ est bien borne critique simple, c.q.f.d.

6.2.2. Les résultats du paragraphe $(6.1)$ donnent successivement :

$$
\begin{gathered}
D_{1}\left(x, y, z_{0}\right)=\frac{\varphi(x) \bar{\psi}(y)}{D_{1}\left(z_{0}, z_{0}, z_{0}\right)} \\
\omega\left(x, z_{0}\right)=\omega\left(z_{0}, z_{0}\right) \frac{\phi(x)}{D_{1}\left(z_{0}, z_{0}, z_{0}\right)} \\
\omega^{\prime}\left(y, z_{0}\right)=\omega^{\prime}\left(z_{0}, z_{0}\right) \frac{\bar{\Psi}(y)}{\bar{D}_{1}\left(z_{0}, z_{0}, z_{0}\right)}
\end{gathered}
$$


Les conditions d'existence d'une solution particulière pour les équations $E\left(z_{0}\right)$ et $E^{\prime}\left(z_{0}\right)$ s'écrivent respectivement :

$$
\omega\left(z_{0}, z_{0}\right) \quad \text { et } \quad \omega^{\prime}\left(z_{0}, z_{0}\right)=0
$$

Si elles sont vérifiées, on en déduit

pour tout $(x, y) \in \Delta_{2}$.

$$
\omega\left(x, z_{0}\right)=\omega^{\prime}\left(y, z_{0}\right)=0
$$

Les solutions particulières sont données respectivement par :

$$
\begin{aligned}
& S(x)=\frac{\Omega_{2}\left(x, z_{0}, z_{0}, z_{0}\right)}{D_{1}\left(z_{0}, z_{0}, z_{0}\right)} \\
& S^{\prime}(y)=\frac{\Omega_{2}^{\prime}\left(y, z_{0}, z_{0}, z_{0}\right)}{\bar{D}_{1}\left(z_{0}, z_{0}, z_{0}\right)}
\end{aligned}
$$

6.2.3. On sait que, pour $z \neq z_{0}$ et voisin de $z_{0}$, la solution unique de $E(z)$ est $\tau(x, z)=\frac{\omega(x, z)}{d(z)}$

Étudions le comportement de $\tau(x, z)$ quand $z$ tend vers $z_{0}$.

En appliquant la formule des accroissements finis à $\omega$ et $d$, on obtient :

$$
\begin{aligned}
\omega\left(x, z_{0}+h\right) & =\omega\left(x, z_{0}\right)-\lambda h \Omega_{2}\left(x, z_{0}+\theta_{1} h, z_{0}+\theta_{1} h, z_{0}+\theta_{1} h\right) \\
d\left(z_{0}+h\right) & =-\lambda h D_{1}\left(z_{0}+\theta_{2} h z_{0}+\theta_{2} h, z_{0}+\theta_{2} h\right)
\end{aligned}
$$

avec

$$
0<\theta_{1}=\theta_{1}(x)<1 \quad \text { et } \quad 0<\theta_{2}<1 .
$$

Si la condition de compatibilité (27) est satisfaite, on a :

et on en déduit :

$$
\forall x \in \Delta_{1}: \tau\left(x, z_{0}+h\right)=\frac{\Omega_{2}\left(x, z_{0}+\theta_{1} h, z_{0}+\theta_{1} h, z_{0}+\theta_{1} h\right)}{D_{1}\left(z_{0}+\theta_{2} h, z_{0}+\theta_{2} h, z_{0}+\theta_{2} h\right)}
$$

$$
\forall x \in \Delta_{1}: \lim _{z \rightarrow z_{0}} \tau(x, z)=S(x)
$$

(On montrerait de même que si $\omega^{\prime}\left(z_{0}, z_{0}\right)=0, \lim _{z \rightarrow z_{0}} \tau^{\prime}\left(y, z_{0}\right)=S^{\prime}(y)$ ). Si la condition $\omega\left(z_{0}, z_{0}\right)=0$ n'est pas satisfaite, le résultat ci-dessus n'est valable qu'aux points $x$ de $\Delta_{1}$ où $\omega\left(x, z_{0}\right)$ s'annule, c'est-à-dire en vertu de (26), sur l'ensemble des zéros de la fonction singulière $\phi(x)$ contenus dans $\Delta_{1}$.

Résumons ces résultats dans le :

THÉORÈME $7:$ Si $z_{0}$ est borne critique simple et si la condition de compatibilité est vérifiée, la limite uniforme, quand $z$ tend vers $z_{0}$, de la solution de l'équation $E(z)$ est une solution particulière de l'équation $E\left(z_{0}\right)$. 


\subsubsection{Système différentiel donnant la solution au voisinage d'une borne cri- tique simple}

Compte tenu de la relation suivante (déduite de (9) avec $n=3, p=1$ et $\phi=K)$ :

$D_{1}\left(x_{1}, y_{1}, z\right), D_{3}\left(x, x_{1}, x_{2}, y, y_{1}, y_{2}, z\right)$

$$
=\left|\begin{array}{ll}
D_{2}\left(x, x_{1}, y, y_{1}, z\right) & D_{2}\left(x, x_{1}, y_{1}, y_{2}, z\right) \\
D_{2}\left(x_{1}, x_{2}, y, y_{1}, z\right) & D_{2}\left(x_{1}, x_{2}, y_{1}, y_{2}, z\right)
\end{array}\right|
$$

de

$$
\frac{\partial}{\partial z} D_{2}\left(x, x_{1}, y, y_{1}, z\right)=-\lambda D_{3}\left(x, x_{1}, z, y, y_{1}, z, z\right)
$$

et du fait que la fonction $z \mapsto D_{1}(z, z, z)$ ne s'annule pas au voisinage de $z_{0}$, on obtient

$\frac{\partial}{\partial z} D_{2}\left(x_{1}, x_{2}, y_{1}, y_{2}, z\right)=\frac{\lambda}{D_{1}(z, z, z)}\left|\begin{array}{ll}D_{2}\left(x_{1}, z, y_{2}, z, z\right) & D_{2}\left(x_{1}, z, y_{1}, z, z\right) \\ D_{2}\left(x_{2}, z, y_{2}, z, z\right) & D_{2}\left(x_{2}, z, y_{1}, z, z\right)\end{array}\right|$

De même, en appliquant (9) à $\Omega_{3}$, on obtient :

$$
\frac{\partial}{\partial z} \Omega_{2}\left(x, x_{1}, y_{1}, z\right)=\frac{\lambda}{D_{1}(z, z, z)}\left|\begin{array}{ll}
D_{2}\left(x, z, y_{1}, z, z\right) & \Omega_{2}(x, z, z, z) \\
D_{2}\left(x_{1}, z, y_{1}, z, z\right) & \Omega_{2}\left(x_{1}, z, z, z\right)
\end{array}\right|
$$

Remarque : Posons $\hat{D}_{2}(x, y, t, z)=D_{2}(x, t, y, t, z)$, l'équation (29) devient alors :

$$
\frac{\partial}{\partial z} \hat{D}_{2}(x, y, t, z)=\frac{\lambda}{D_{1}(z, z, z)}\left|\begin{array}{ll}
\hat{D}_{2}(x, t, z, z) & \hat{D}_{2}(x, y, z, z) \\
\hat{D}_{2}(t, t, z, z) & \hat{D}_{2}(t, y, z, z)
\end{array}\right|
$$

De même, en posant $\hat{\Omega}_{2}(x, t, z)=\Omega_{2}(x, t, t, z)$, l'équation (30) devient :

$$
\frac{\partial}{\partial z} \hat{\Omega}_{2}(x, t, z)=\frac{\lambda}{D_{1}(z, z, z)}\left|\begin{array}{ll}
\hat{D}_{2}(x, t, t, z) & \hat{\Omega}_{2}(x, z, z) \\
\hat{D}_{2}(t, t, z, z) & \hat{\Omega}_{2}(t, z, z)
\end{array}\right|
$$

Au lieu du système $\left(S F_{0}\right)$ qui n'est plus utilisable au voisinage de la borne critique $z_{0}$, on intègre le système $\left(S F_{1}\right)$ formé des équations $(29 \mathrm{bis}),(30 \mathrm{bis})$ et :

$$
\begin{gathered}
d^{\prime}(z)=-\lambda D_{1}(z, z, z) \\
\frac{\partial}{\partial z} D_{1}(x, y, z)=-\lambda D_{2}(x, z, y, z, z)=-\lambda \hat{D}_{2}(x, y, z, z) \\
\frac{\partial}{\partial z} \omega(x, z)=-\lambda \Omega_{2}(x, z, z, z)=-\lambda \hat{\Omega}_{2}(x, z, z) .
\end{gathered}
$$


En pratique, il suffit de connaitre $d, D_{1}$ et $\hat{D}_{2}$ pour calculer $\omega$ et $\Omega_{2}$ au moyen de (13) et $(8)(n=2, \phi=f)$.

Considérons maintenant un intervalle $I_{\alpha}=\left[z_{0}-\alpha, z_{0}+\alpha\right]$ sur lequel $\left|D_{1}(z, z, z)\right| \geqslant k_{1}>0$. Sur cet intervalle, le système $\left(S F_{1}\right)$ ci-dessus admet au moins comme solution le quintuplet $\left(d, D_{1}, d_{2}, \omega, \Omega_{2}\right)$ des fonctions à partir desquelles on l'a construit.

Notons $E_{1}\left[z_{0}-\alpha, z_{0}+\alpha\right]$ l'ensemble des quintuplets $\left(\delta, \mathscr{D}_{1}, \mathscr{D}_{2}, \bar{\Omega}, \bar{\Omega}\right)$ de fonctions continues respectivement sur $I_{\alpha}, \Delta_{2} \times I_{\alpha}, \Delta_{4} \times I_{\alpha}, \Delta_{1} \times I_{\alpha}$ et $\Delta_{3} \times I_{\alpha}$, possédant chacune une dérivée partielle en $z$ globalement continue sur son domaine de définition, vérifiant

$\delta\left(z_{0}-\alpha\right)=d\left(z_{0}-\alpha\right), \ldots, \bar{\Omega}_{2}\left(x_{1}, x_{2}, y_{1}, z_{0}-\alpha\right)=\Omega_{2}\left(x_{1}, x_{2}, y_{1}, z_{0}-\alpha\right)$ et $\left|\mathscr{D}_{1}(z, z, z)\right| \geqslant K_{1}>0$ (la constante $K_{1}$ dépendant du quintuplet). Dans ces conditions, on peut énoncer le :

THÉORÈME $8:$ Le système $\left(S F_{1}\right)$ admet comme solution unique dans $E_{1}\left(I_{\alpha}\right)$ le quintuplet $\left(d, D_{1}, D_{2}, \omega, \Omega_{2}\right)$.

Démonstration : Elle est analogue à celle du théorème 4. Les seconds membres du système $\left(S F_{1}\right)$ vérifient localement une condition de Lipschitz, d'où l'unicité de la solution.

6.3. Cas où $z_{0}$ n'est pas racine simple de $d(z)=0$

S'il existe $n \in \mathbf{N}$ tel que

$$
\left.\check{\circ}_{n}=D_{n}\left(\stackrel{\circ}{Z}_{n}, \stackrel{\circ}{Z}_{n}, z_{0}\right) \neq 0 \quad \text { (en posant } \stackrel{\circ}{Z}_{n}=\left(z_{0}, z_{0}, \ldots, z_{0}\right) \in \Delta_{n}\right),
$$

on établit, au moyen du théorème 1 et des relations (6), (7), (8) et (9), un système $\left(S F_{n}\right)$ que l'on intègre sur un intervalle où $\left|D_{n}\left(Z_{n}, Z_{n}, z_{0}\right)\right| \geqslant k_{n}>0$. Mais, naturellement, l'intérêt est moins grand pour le calcul numérique.

\section{EXEMPLE}

Étudions l'équation :

$$
E(z) \tau(x, z)=e^{2 x}+\frac{3}{2} \int_{0}^{z} e^{x-s} \tau(s, z) d s \quad(0 \leqslant z \leqslant 1)
$$

On obtient facilement :

$$
\begin{gathered}
d(z)=1-\frac{3}{2} z \quad, \text { d'où } z_{0}=\frac{2}{3} \quad \text { (seule borne critique) } \\
D_{1}(x, y, z)=e^{x-y}>0 \quad ; \quad D_{2} \equiv 0 \\
\omega(x, z)=\left(1-\frac{3}{2} z\right) e^{2 x}+\frac{3}{2} e^{x}\left(e^{z}-1\right)
\end{gathered}
$$


Remarquons que

$$
\omega\left(z_{0}, z_{0}\right)=\frac{3}{2} e^{\frac{2}{3}}\left(e^{\frac{2}{3}}-1\right) \neq 0
$$

donc la condition de compatibilité n'est pas vérifiée, et $\tau(x, z)=\omega(x, z) / d(z)$ tend vers $+\infty$ quand $z \rightarrow \frac{2}{3}$ par valeurs inférieures et vers $-\infty$ quand $z \rightarrow \frac{2}{3}$ par valeurs supérieures (ce que confirment les résultats numériques ci-dessous). Enfin, on peut calculer également

$$
\begin{aligned}
\Omega_{2}\left(x, x_{1}, y_{1}, z\right) & =e^{2 x} e^{x_{1}-y_{1}}-e^{2 x_{1}} e^{x-y_{1}} \\
& =e^{x+x_{1}-y_{1}}\left(e^{x}-e^{x_{1}}\right)
\end{aligned}
$$

On constate bien que

$$
\frac{\partial}{\partial z} \omega(x, z)=-\frac{3}{2} \Omega_{2}(x, z, z, z)
$$

Pour le calcul numérique de la solution, on partage $[0,1]$ en huit sous-intervalles égaux et on intègre le système $\left(S F_{0}\right)$ au moyen de la méthode classique de Runge-Kutta de rang 4. (On n'a pas utilisé le système $\left(S F_{1}\right)$ au voisinage de $z_{0}=\frac{2}{3}$, le pas $h=0.125$ étant assez grand pour ne pas provoquer d'erreurs importantes au voisinage de $\left.z_{0}\right)$. Si $\bar{\tau}(x, z)$ désigne la valeur calculée de la solution $\tau(x, z)$ de $E(z)$, on donne dans le tableau ci-dessous quelques valeurs de $\bar{\tau}(1, z)$ et de $\bar{e}(z)=10^{7} x(\tau(1, z)-\bar{\tau}(1, z))$.

\begin{tabular}{|c|c|c|c|c|c|c|c|c|}
\hline$z$ & 0,125 & 0,25 & 0,375 & 0,5 & 0,625 & 0,75 & 0,875 & 1 \\
\hline $\bar{\tau}(1, z)$ & 0,805 & 0,824 & 11,629 & 17,963 & 64,032 & $-29,046$ & $-10,863$ & $-6,623$ \\
\hline $\bar{e}(z)$ & $-0,57$ & $-1,57$ & $-3,59$ & $-8,96$ & -48 & $+30,87$ & $+15,46$ & $+11,87$ \\
\hline
\end{tabular}

\section{BIBLIOGRAPHIE}

1. K. E. AtKinson. The Numerical Solution of Fredholm Integral Equations of the second kmd. Stam J. Numer. Anal. 4. 1967. p. 237-248.

2. F. R. Gantmacher, The Theory of Matrices, Vol. 1, Chelsea, New York, 1959.

3. I. C. Gohberg et M. G. KreIN, Theory and Applications of Volterra Operators in Hilbert Space, A.M.S. Vol. 24, 1970.

4. E. Goursat, Cours d'Analyse Mathématique, Tome 3, Gauthier-Villars, Paris, 1927.

5. P. POUZET, Méthodes numériques par pas pour le traitement de certaines équations intégrales linéaires de Fredholm. Colloque d'Analyse Numérique, Aussois, 1969. 
6 P POUZET et C Broudiscou, Trattement numérique des équations intégrales linéaires de Fredholm a resolvante partielle continue, C $\mathrm{R}$ Ac Sc Paris, t 268, (1969), A $1279-1281$

7 F Riesz et B Sz NAGY, Leçons d'Analyse Fonctionnelle, Gauthier-Villars, Parı, 1968

8 P SABLONNIÈRE, Utılisation des déterminants de Fredholm dans la résolution numérlque des équations intégrales lineaires à noyau contınu Colloque d'Analyse numénque, Gourette, 1974 C R A Sc, Par1s, t 279, (1974), A 337-340

9 A SCHUMITZKY et T WENSKA Imbedding a class of linear integral equations through the first criticat , oint Siam J Math Anal Vol $4 \mathrm{n}^{\circ} 4$ nov 73 p 592-69)8

10 F Smithies Integral Equations Cambridge University Press 1958

11 S L SOBOLEv, Remarks on the Numerical solution of integral equations lzv Akad Nauk S S S R Ser Mat 201956 p 413-435 